\title{
Thermographic Laser Doppler Velocimetry
}

\author{
Anthony O. Ojo, ${ }^{1}$ Benoit Fond, ${ }^{1}$ Berend G. M. Van Wachem, ${ }^{1}$ \\ Andrew L. Heyes, ${ }^{2}$ ANd Frank Beyrau ${ }^{3 *}$
}

\author{
${ }^{1}$ Department of Mechanical Engineering, Imperial College London, London SW7 2AZ, UK \\ ${ }^{2}$ Department of Civil Engineering, University of Leeds, Leeds LS2 9JT, UK \\ ${ }^{3}$ Lehrstuhl für Technische Thermodynamik, Otto-von-Guericke-Universität Magdeburg, 39106 Magdeburg, Germany \\ *Corresponding author: frank.beyrau@ovgu.de
}

Received XX Month XXXX; revised XX Month, XXXX; accepted XX Month XXXX; posted XX Month XXXX (Doc. ID XXXXX); published XX Month XXXX

\begin{abstract}
We propose a point-measurement technique for simultaneous gas temperature and velocity measurement based on thermographic phosphor particles dispersed in the fluid. The flow velocity is determined from the frequency of light scattered by $\mathrm{BaMgAl}_{10} \mathrm{O}_{17}: \mathrm{Eu}^{2+}$ phosphor particles traversing the fringes like in conventional LDV. Flow temperatures are derived using a two-colour ratio method applied to the phosphorescence from the same particles. This combined diagnostic technique is demonstrated with a temperature precision of $4-10 \%$ in a heated air jet during steady operation for flow temperatures up to $624 \mathrm{~K}$. The technique provides correlated vector-scalar data at high spatial and temporal resolution. (C) 2015 Optical Society of America
\end{abstract}

OCIS codes: (120.1740) Combustion diagnostics, (280.0280) Remote sensing and sensors, (280.2490) Flow diagnostics, (280.3340) Laser Doppler velocimetry, (280.6780) Temperature, (160.2540) Fluorescent and luminescent materials.

http://dx.doi.org/10.1364/OL.99.099999

In flow processes characterised by thermal mixing, heat and momentum transport are greatly influenced by turbulence due to the dynamics of coherent structures inherent in such flows [1]. The prevailing coupling between the temperature-velocity flow fields are reflected as turbulent heat fluxes. Therefore, simultaneous measurements of these flow quantities are needed to provide better understanding of the flow mechanisms and to further enhance the design and optimisation of the associated industrial flow equipment.

Measurements of local flow velocities and their fluctuations have been made possible through the well-established laser Doppler velocimetry (LDV) technique [2], which was first demonstrated almost 51 years ago [3]. LDV provides time-resolved point measurements with a high accuracy and about $1 \%$ theoretical error [4]. With probe volume diameters typically in the order of 50-200 $\mu \mathrm{m}$ [5], LDV also offers high spatial resolutions and is suitable for flow measurement in the vicinity of walls [2], and where there is restricted optical access [6]. In order to perform joint velocity and temperature measurements, this technique has been used simultaneously with other optical techniques. In the joint diagnostic approach, temperature measurement was achieved by Raman-Rayleigh spectroscopy [7] or coherent anti-Stokes Raman spectroscopy (CARS) [8], which are mature thermometry techniques. However, these joint measurement techniques involve very high costs and experimental complexities. Furthermore, in the combined technique, because the sampling time of velocity and temperature differs, knowledge of the lag time between them is required for measurement synchronization. Therefore, additional complications arise, which must be solved prior to accurate evaluation of the heat fluxes [8].

The use of a flow tracer particle having temperature-dependent properties would allow simultaneous point measurements of flow temperature and velocity. In this case, the joint measuring system would consist of a simple experimental setup, where sampling of these gas properties are spatially and temporally correlated. Thermographic phosphor particles, which are optically active ceramic materials with temperature dependent luminescent properties, are suitable tracer materials for this concept. The use of these particles is based on the fundamental principle that they effectively trace the flow motion [9] and temperature [10] without altering the fluid properties.

Recently, the optical properties of thermographic phosphor particles, entrained in fluid flows, have been exploited to provide correlated planar information on flow velocity and temperature [1012] in an approach termed thermographic particle image velocimetry. There, the conventional particle image velocimetry (PIV) technique is used to visualize the velocity field by recording elastically scattered laser light from particles entrained in the flow. Simultaneously, the recorded phosphorescence emission that follows UV excitation of the same particles, allows the measurement of the temperature field based on a two-colour ratio approach. Recently, joint high-speed planar measurements using this technique have been demonstrated using multi-kHz repetition rate lasers and cameras [13]. However, these planar measurements are typically performed at spatial resolution above $400 \mu \mathrm{m}$.

In this letter, we propose a novel point measurement technique for remote gas thermometry and velocimetry at a high spatial and temporal resolution. It is applied to a heated jet of air being discharged into the ambient by probing optical signals from single $\mathrm{BaMgAl}_{10} \mathrm{O}_{17}: \mathrm{Eu}^{2+}$ (BAM:Eu) phosphor particles seeded into the flow. For these measurement, $2 \mu \mathrm{m}$ BAM:Eu particles (KEMK63/UF-P1, Phosphor Technology) were used. The high luminescence yield of BAM:Eu, apparent from the bright blue emission after UV excitation, and the short lifetime of about $1 \mu \mathrm{s}$ at room temperature, makes it useful for our proposed technique [14]. This phosphorescence emission occurs due to transition between $4 \mathrm{f}^{6} 5 \mathrm{~d}^{1} \rightarrow 4 \mathrm{f}^{7}$ energy levels of the activator ion, $\mathrm{Eu}^{2+}[15]$, and is temperature dependent [16]. One of our recent studies showed that neither the emission intensity nor the spectral response of this phosphor show any dependence to oxygen concentration over the range investigated $\left(0-200 \mathrm{mbar}_{2}\right.$ partial 
pressure up to $920 \mathrm{~K}$ ) and that the phosphor is suitable for measurements over that range [17]. Furthermore, the spectral response of BAM:Eu was shown to be independent of pressure up to $10 \mathrm{kbar}$ [18].

We exploited the temperature dependence of the radiative emission of single BAM:Eu particles to evaluate the flow temperature using the spectral intensity ratio method. Figure 1 shows normalised emission spectra at different temperatures superimposed with the transmission profiles of appropriate filter combinations used to segregate two spectral regions for intensity ratio analysis. The flow velocity is simultaneously evaluated from the frequency of the Doppler burst produced by the same particle which crosses the fringes in the measurement volume of a conventional LDV arrangement.

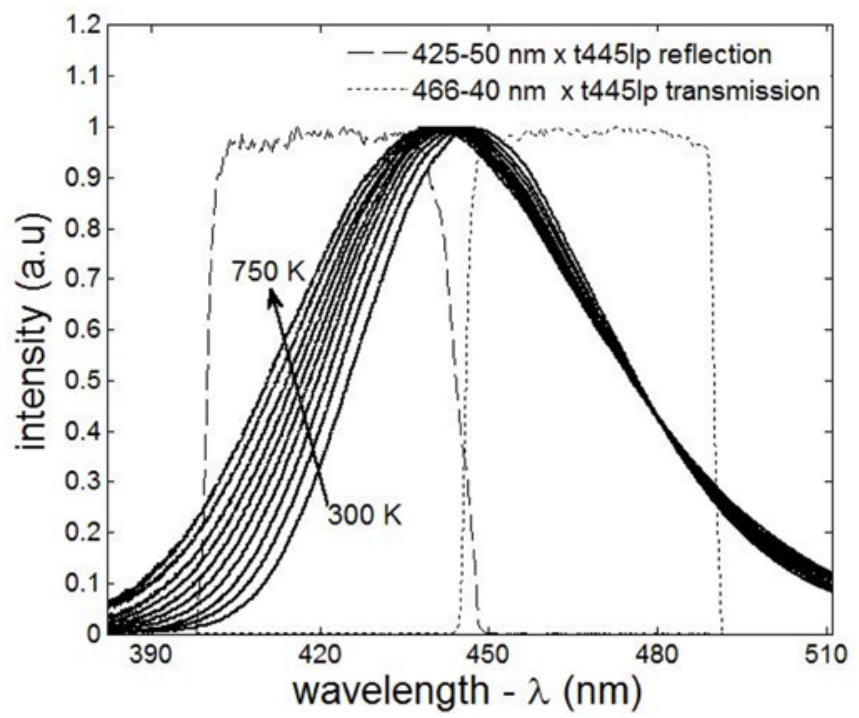

Fig. 1. Normalised emission spectra of BAM:Eu with superimposed transmission profiles of the two filter combinations.

Our experimental setup is illustrated in Fig. 2. As shown, a one component LDV system in its most simple form is built from two laser beams ( $514.5 \mathrm{~nm}$, from an Argon Ion laser), separated by a distance of $36 \mathrm{~mm}$ and focused by a lens ( $\mathrm{f}=250 \mathrm{~mm}$ ) to form a measuring volume at the intersection of the beams. For demonstration, we restrict ourselves to this simple LDV set-up, where we do not correct for directional ambiguity in velocity measurements. A third beam from a $375 \mathrm{~nm}, 70 \mathrm{~mW}$ UV diode laser (PhoxX - Omicron Lasers) is directed and superimposed on the LDV measuring volume using the same focusing lens. The LDV measuring volume and the matched UV beam in the focus were measured to have a $1 / \mathrm{e}^{2}$ beam diameter of about 150 $\mu \mathrm{m}$. To monitor the overlap of the three beams, a glass window with an anti-reflective coating on the back-side was placed after the focusing lens, reflecting a portion of the beams, towards a CCD mounted on a translation stage, itself equipped with two OD 3.0 Neutral density filters. We are using a colour camera with a Bayer pattern for our beam view system. When looking at the blue and green channels separately, the three beams can be overlapped perfectly, and the details of the fringe pattern can be clearly monitored [19].

Prior to use, the particles were treated with $\mathrm{SiO}_{2}$ coating to reduce inter-particle forces by cancelling surface charge, and then dried for 4 hours at $393 \mathrm{~K}$ in an oven. These particles were subsequently seeded in the flow using an in-house built magnetic stirrer particle seeder, which was grounded to prevent build-up of static charges.

We used a $85 \mathrm{~mm}$ (f/1.4D) Nikon camera lens to create a single optical detection path through which Mie scattered light and phosphorescence emission from the probe volume were focused through a $200 \mu \mathrm{m}$ slit. In this experiment the magnification is 1 . Using a $50 \mu \mathrm{m}$ pinhole, positioned at the measurement volume and in front of a stable tungsten halogen light source, we performed a collection efficiency test by translating both the pinhole and light source in the cross-stream direction. The results of this test showed that the effective length of the measurement volume and the minimum spot size created by the lens in the image plane is $250 \mu \mathrm{m}$ and $150 \mu \mathrm{m}$ respectively $\left(1 / \mathrm{e}^{2}\right.$ width). With the streamwise and spanwise spatial resolution being both defined by the beam diameter $(150 \mu \mathrm{m})$, we conclude that the spatial resolution of our measurement is $250 \mu \mathrm{m}$ x $150 \mu \mathrm{m} \times 150 \mu \mathrm{m}$. The optical signals were collected from the slit and collimated by a plano convex lens, $\mathrm{f}=75 \mathrm{~mm}$. The collimated light was spectrally separated by a long pass dichroic beam splitter (Chroma - t500lpxrxtUF1) which transmits the Doppler bursts, and reflects the phosphorescence light. The phosphorescence light was then spectrally separated using a combination of a long pass dichroic beam splitter (Chroma - t445lp) and a pair of interference filters (Edmund Optics: 425-50 nm, and 466-40 nm (notation is CWL-FWHM)). To remove any remaining scattered $375 \mathrm{~nm}$ light, a $400 \mathrm{~nm}$ long pass coloured glass filter was placed in front of the 4 455lp. We verified experimentally that contributions from scattered $375 \mathrm{~nm} / 514 \mathrm{~nm}$ laser light to the phosphorescence signal are negligible due to the high transmittance/reflectance of the long pass filters within the bandpass, and the high attenuation of the interference filters outside the bandpass.

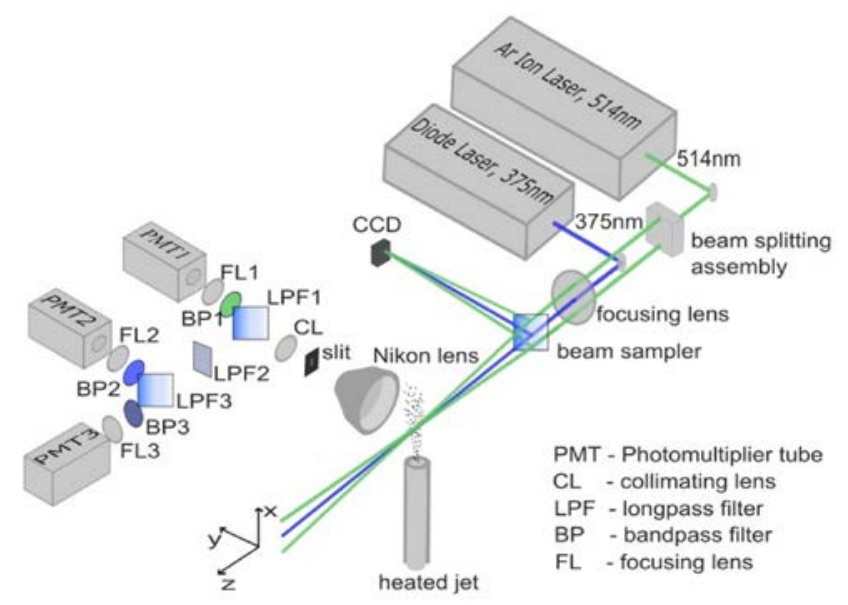

Fig. 2. Experimental setup for simultaneous measurements. LPF1: 500 nm, LPF2: 400 nm, LPF3: 445 nm, BP1: 514-10 nm, BP2: 425-50 nm, BP3: $466-40 \mathrm{~nm}$, FL1, 2, and 3: focal length $=50 \mathrm{~mm}$.

A Photomultiplier Tube (PMT) (Thorlabs), hereafter referred to as PMT1, fitted with a focusing lens ( $\mathrm{f}=50 \mathrm{~mm}$,) and a 514-10 nm filter was used to acquire the Doppler bursts from the scattered $514 \mathrm{~nm}$ light. Two other identical PMTs (Hamamatsu R955HA - PMT2 and PMT3) fitted with focusing lenses ( $\mathrm{f}=50 \mathrm{~mm}$ ) each received the phosphorescence photons from the respective spectral regions (PMT2-425 nm, and PMT3-466 nm). These phosphorescence detecting PMTs were operated at the same supply voltage of $500 \mathrm{~V}$ (gain $10^{5}$ ), and were experimentally confirmed to have output pulse linearity $<2 \%$ for PMT output currents $<1 \mathrm{~mA}$. This was in agreement with the manufacturer's specification [20]. The outputs of the three PMTs were fed into three channels of a Tektronix DPO4054 oscilloscope to provide digitization of the signals. The electrical output from PMT1 was terminated by the $50 \Omega$ impedance on the oscilloscope to preserve the temporal resolution of the Doppler burst signals. The output voltages resulting from the spectrally separated signals from PMT2 and PMT3, were measured using a variable terminator (Thorlabs) to give an actual resistance of $9.9 \mathrm{k} \Omega$. Waveforms of these three signals were acquired by the oscilloscope over a $400 \mathrm{~ms}$ record duration. Background signals were also acquired in the same manner but without laser illumination. 
The waveforms consist of random pulses of varying intensities and widths, due to the random arrival of particles in the measurement volume. The waveform of signals from PMT1 contains Doppler bursts having a Gaussian envelope. Similarly, the waveform of signals from the two other PMTs consists of near Gaussian phosphorescence bursts, which are simultaneous with the Doppler bursts. We expected these Gaussian bursts from a luminescent particle since the intensity distribution profile of the UV beam is near Gaussian $\left(\mathrm{M}^{2}<1.2\right)$, and the phosphorescence decay time of the particles is short (around $1 \mu \mathrm{s}$ at room temperature) compared to the transit time. Figure 3 shows an illustration of temporally correlated Mie-scattering (Doppler) and phosphorescence bursts recorded at a flow temperature of $435 \mathrm{~K}$ and velocity of $3.08 \mathrm{~m} / \mathrm{s}$. The intensities of these bursts follow the analytical expressions;

$$
\begin{gathered}
I_{L D V}=I_{A} e^{-2\left(t / t_{b}\right)^{2}}(1+(m \cdot \cos (2 \pi f t))) \\
I_{466 n m}=I_{B} e^{-2\left(t / t_{b}\right)^{2}} \\
I_{425 n m}=R \cdot I_{B} e^{-2\left(t / t_{b}\right)^{2}}
\end{gathered}
$$

where $R$ is the expected ratio $=I_{425} \mathrm{~nm} / I_{466} \mathrm{~nm}, t_{b}$ is half signal duration $\left(1 / \mathrm{e}^{2}-\right.$ intensity limit), $f=$ Doppler frequency, $m=$ modulation depth, $I_{A}$ and $I_{B}$ are the amplitude of the LDV and phosphorescence bursts signals respectively.

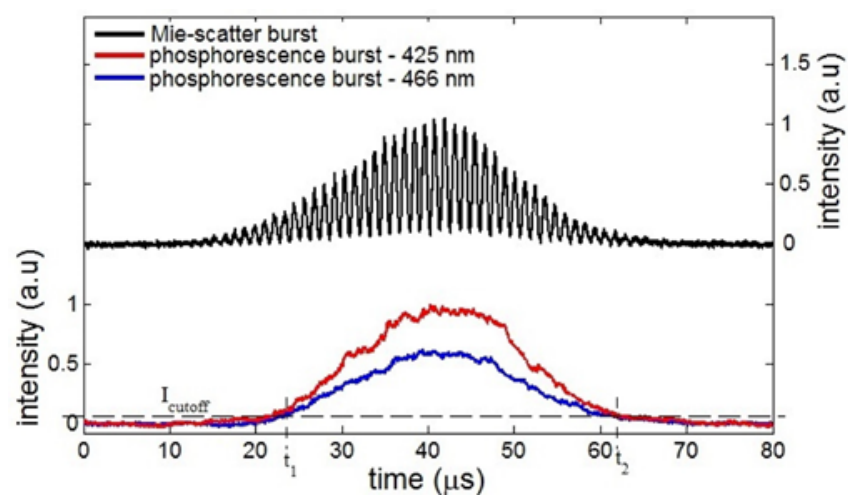

Fig. 3. Signal from a BAM:Eu particle simultaneously producing a Miescattering (Doppler) and phosphorescence bursts.

From our measurements at room temperature, the mean peak output current is $6.3 \mu \mathrm{A}$ from PMT3 (466 nm channel), and for a single burst from a particle with transit time $\left(\mathrm{t}_{\mathrm{b}}\right)$ of $87.2 \mu \mathrm{s}$, an estimate of about $2.5 \times 10^{5}$ photons are detected. We see that the probability of PMT photocathode saturation is negligibly small. We infer this since the number of incident photons which will cause saturation for the same duration of burst is estimated as (itb/eq) $3.5 \times 10^{8}$ photons, where $i$ is the photocathode current chosen here as $0.1 \mu \mathrm{A}$ (specified upper limit of $10 \mu \mathrm{A}$ [20]), $e$ is the charge of an electron, and $\eta$ is the quantum efficiency of the photocathode material.

The acquired waveforms were processed in Matlab. The mean background intensity was subtracted from the respective waveforms that contained phosphorescence signals. In order to identify the bursts in the subtracted waveforms, a cutoff filter set at $5 \mathrm{mV}$ was applied on one phosphorescence channel, and a time window was created for each burst based on this $5 \mathrm{mV}$ (I cutoff) threshold as illustrated on Fig. 3 . This time window allows each burst signal from the other channels to be identified and isolated. The number of bursts is evaluated as an indication of the average particle seeding concentration for each acquisition. It was found to be in the range $10^{10}-10^{11}$ particles per $\mathrm{m}^{3}$.
This seeding density is appropriate for LDV because the Doppler burst signals provided a good Signal to Noise Ratio (SNR) based on their evaluated frequency content. At a particle concentration of $1.2 \times 10^{11}$ particles per $\mathrm{m}^{3}, 1,647$ bursts were obtained on average by our recording sequence ( $400 \mathrm{~ms}$ duration) at room temperature, and at a mean flow velocity of $1.72 \mathrm{~m} / \mathrm{s}$. In theory, consecutive burst signals with interarrival times in the order of $40 \mu \mathrm{s}$ can be measured. With burst duration as short as $40 \mu \mathrm{s}$, which is on the order of the particle response time, this technique provides high temporal resolution.

The flow velocity was obtained by applying a FFT algorithm on each Doppler burst within each time window. Within the individual time window, sufficient numbers of fringes $(>25)$ were available to evaluate the Doppler frequency and minimize the error due to finite transit time broadening. Only Doppler bursts having SNR $>7.5$ were considered for further analysis.

The flow temperature was obtained by analyzing the corresponding phosphorescence bursts on the background-subtracted traces. The intensity of these bursts, which represents optical signals from the 425-50 nm and 466-40 nm spectral regions respectively, was integrated within the Full Width at Half Maximum (FWHM) for each burst. A cutoff of $0.5 \times 10^{-6}$ V.s was then applied to the time integrated intensities in order to reject signals which create noisy intensity ratios. This cutoff was defined based on measurements where a pulse LED light was imaged on PMT2 and PMT3 with the same gain settings used in the flow measurements. From this LED experiments, the cutoff of 0.5 $x 10^{-6}$ V.s resulted in a normalized ratio precision of $6 \%$. The time integrated intensities surviving the applied cut off were used to obtain a compilation of ratios $\left(\mathrm{I}_{425 \mathrm{~nm}} / \mathrm{I}_{466} \mathrm{~nm}\right)$. These ratios were converted to temperatures using a curve fit from calibration data of ratio as a function of temperature.

The ratio calibration was performed in an electrically heated jet. During a steady jet operation, measurements were taken in the jet potential core, along the jet centreline, and at a position $3 \mathrm{~mm}$ above the jet. A sufficient number of independent measurements $(>500)$ obtained at each temperature was used to calculate the mean intensity ratio. The jet exit temperature was monitored with a K-type thermocouple. The ratio versus temperature curve is shown in Fig. 4, where a quadratic curve is applied to these in-flow calibration data and subsequently used to convert the ratio to temperature. Data obtained from calibration sequences carried out at different days confirmed the repeatability of the calibration procedure within $2.2 \%$. For the filter combination employed in this work, the ratio sensitivity to temperature is seen to decrease as the temperature increases. We establish that the sensitivity falls from $0.29 \% / \mathrm{K}$ to $0.075 \% / \mathrm{K}$, for the calibration temperature range covered.

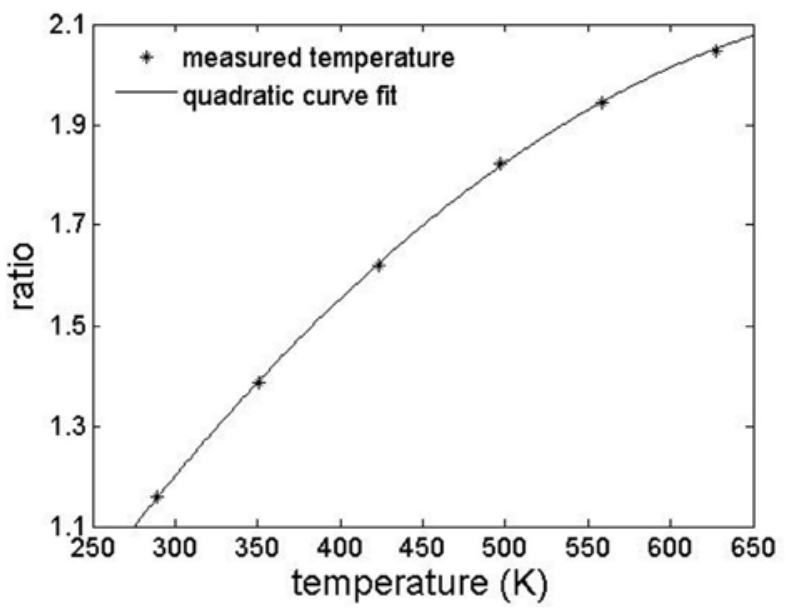

Fig. 4. Calibrated ratio as a function of gas temperature. 
Figure 5 shows the histograms which were compiled using the statistics obtained from measurements taken in the potential core of the heated jet at four steady jet exit temperatures. Correction for velocity bias associated with the LDV technique was implemented using the residence time weighing approach and mean flow velocities of $1.72 \mathrm{~m} / \mathrm{s}, 2.51 \mathrm{~m} / \mathrm{s}, 2.93 \mathrm{~m} / \mathrm{s}$ and $3.74 \mathrm{~m} / \mathrm{s}$ were realized at these temperatures $(293 \mathrm{~K}, 425 \mathrm{~K}, 505 \mathrm{~K}$ and $624 \mathrm{~K}$ respectively). The evaluated turbulence intensities $(1.25 \%, 1.13 \%, 1.21 \%$ and $1.22 \%$ respectively) at these flow velocities confirm the steady nature of the heated jet. The single shot precision (1 standard deviation) of the thermometry technique was evaluated and is noted on Fig. 5. At room temperature, the standard deviation is about $12 \mathrm{~K}$, but due to a decrease in both SNR and temperature sensitivity as temperature increases, the deviation at $624 \mathrm{~K}$ is $63 \mathrm{~K}$. A comparison with the pulsed LED light test mentioned above showed that the error is mainly limited by random uncertainties, which are inversely proportional to the square root of the signal intensity (shot-noise regime). The signal intensity is itself proportional to the power of the laser used in this study, and to the inverse of the flow velocity.

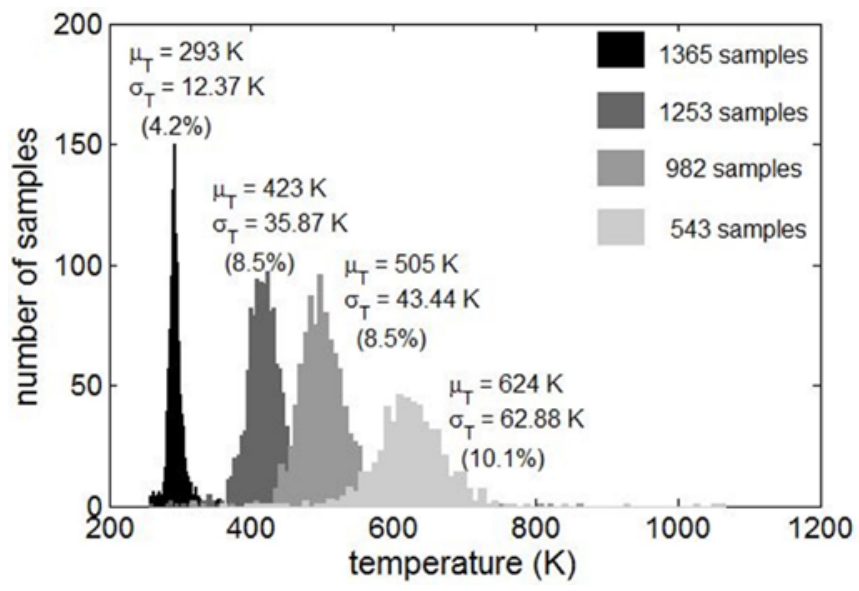

Fig. 5. PDFs for correlated measurements of gas temperature .

In summary, we demonstrated that simultaneous remote measurements of gas temperature and velocity using seeded thermographic phosphor particles can be achieved on a point measurement basis by probing single particles with continuous wave lasers. The results show that time resolved measurements of vectorscalar gas properties with a spatial resolution of $250 \mu \mathrm{m} \times 150 \mu \mathrm{m} \times$ $150 \mu \mathrm{m}$ are possible using a relatively simple experimental setup that requires a single seeded tracer. A precision between 4 and 10\% was obtained for flow temperatures between $293 \mathrm{~K}$ and $624 \mathrm{~K}$. Use of a more powerful laser is expected to significantly improve the technique precision. In addition, there is an almost infinite variety of thermographic phosphors. A tracer with a more pronounced temperature sensitivity could be used for additional improvements. In this context, a recent study showed that $\mathrm{ZnO}$ can be used for high precision flow thermometry at low temperatures [21]. A phosphor which covers a wider temperature range would also be useful in reactive flow applications.

Acknowledgment. We acknowledge financial support of parts of this study by the Nigerian Government through its Presidential Special Scholarship Scheme for Innovation and Development (PRESSID) program

\section{REFERENCES}

1. E. Rathakrishnan, Applied Gas Dynamics, (John Wiley \& Sons 2010), p. 512.

2. H. E. Albrecht, Borys, M., Damaschke, N., and Tropea, C., Laser Doppler and Phase Doppler Measurement Techniques, 1 ed. (Springer, 2002).

3. Y. Yeh and H. Z. Cummins, Appl. Phy. Letters 4, 10. 176 (1964).

4. A.Van Maaren, and L.P.H. de Goey, Comb. Sci. and Tech., 99, 105 (1994).

5. Z. Zhang, LDA Application Methods, (Springer, 2010), p. 45.

6. E. Ergin and M. Ö. Semih, Meas. Sci. Technol. 16. 2038 (2005).

7. R. W. Dibble, V. Hartmann, and R. W. Schefer, Exp. Fluids. 5. 103 (1987).

8. M. J. Tummers, E. H. van Veen, N. George, R. Rodink, and K. Hanjalic, Exp. Fluids. 37.364 (2004).

9. A. Melling, Meas. Sci, Technol. 8. 1406 (1997).

10. B. Fond, C. Abram, A. L. Heyes, A. M. Kempf, and F. Beyrau. Opt. Express, 20, 22118 (2012).

11. A. Omrane, P. Petersson, M. Alden, and M. A. Linne, Appl. Phys. B. 92.99 (2008).

12. N. J. Neal, J. Jordan, and D. Rothamer, SAE Int. J. Engines 6, 300 (2013).

13. C. Abram, B. Fond, A. L. Heyes, and F. Beyrau, Appl. Phys. B. 111. 155 (2013).

14. B. Fond, C. Abram, and F. Beyrau, Appl. Phys. B. 118. 393 (2015).

15. G. Blasse. Phys. Stat. sol. (b) 55. K131 (1973).

16. G. Särner, M. Richter, and M. Alden. Meas. Sci. Technol. 19. 125304 (2008).

17. B. Fond, C. Abram, and F. Beyrau, "Characterisation of the luminescence properties of BAM:Eu particles as a tracer for thermographic particle image velocimetry", submitted to Applied Physics B, May 2015.

18. R. Turos-Matysiak, M. Grinberg, J.W. Wang, W.M. Yen, and R.S. Meltzer. J.Lumin. 122-123 .107 (2007).

19. S. Pfadler, F. Dinkelacker, F. Beyrau, and A. Leipertz. Combust. and Flame. 156. 1552 (2009).

20. Hamamatsu Photonics, Photomultiplier Tubes: Basics and Applications, 3 ed., (2007).

21. C. Abram, B. Fond and F. Beyrau, Opt. Express, 23. 19453 (2015). 


\section{Full References}

1. E. Rathakrishnan, "Applied Gas Dynamics," John Wiley \& Sons, Inc, 2010.

2. H. E. Albrecht, Borys, M., Damaschke, N., and Tropea, C., Laser Doppler and Phase Doppler Measurement Techniques, 1 ed.: Springer, 2002.

3. Y. Yeh and H. Z. Cummins, "Localized Fluid Flow Measurements with an He-Ne Laser Spectrometer," Applied Physics Letters 4, vol. 10, p. 176, 1964.

4. A. Van Maaren and L. P. H. de Goey, "Laser Doppler Thermometry in Flat Flames," Combustion Science and Technology, vol. 99, pp. 105118, 1994/08/01 1994.

5. Zhenji. Zhang, LDA Application Methods, (Springer, 2010), p. 45.

6. E. Ergin and M. Ö. Semih, "A spark-plug LDV probe for in-cylinder flow analysis of production IC engines," Measurement Science and Technology, vol. 16, p. 2038, 2005.

7. R. W. Dibble, V. Hartmann, and R. W. Schefer, "Conditional sampling of velocity and scalars in turbulent flames using simultaneous LDV-Raman scattering," Experiments in Fluids, Springer-Verlag, vol. 5, pp. 103 113, 1987.

8. M. J. Tummers, E. H. van Veen, N. George, R. Rodink, and K. Hanjalic, "Measurement of velocity-temperature correlations in a turbulent diffusion flame," Experiments in Fluids, vol. 37, pp. 364-374, 2004.

9. A. Melling, "Tracer particles and seeding for particle image velocimetry," Measurement Science and Technology., vol. 8, pp. 14061416, 1997.

10. B. Fond, C. Abram, A. L. Heyes, A. M. Kempf, and F. Beyrau, "Simultaneous temperature, mixture fraction and velocity imaging in turbulent flows using thermographic phosphor tracer particles," Optics Express, vol. 20, pp. 22118-22133, Sep 242012.

11. A. Omrane, P. Petersson, M. Alden, and M. A. Linne, "Simultaneous 2D flow velocity and gas temperature measurements using thermographic phosphors," Applied Physics B-Lasers and Optics, vol. 92, pp. 99-102, Jul 2008.

12. N. J. Neal, J. Jordan, and D. Rothamer, "Simultaneous Measurements of In-Cylinder Temperature and Velocity Distribution in a Small-Bore Diesel Engine Using Thermographic Phosphors," SAE International Journal of Engines, pp. 300-318, 2013.

13. C. Abram, B. Fond, A. L. Heyes, and F. Beyrau, "High-speed planar thermometry and velocimetry using thermographic phosphor particles," Applied Physics B, vol. 111, pp. 155-160, 2013/05/01 2013.

14. B. Fond, C. Abram, and F. Beyrau, "On the characterisation of tracer particles for thermographic particle image velocimetry," Applied Physics B, vol. 118, pp. 393-399, 2015/03/01 2015.

15. G. Blasse, "On the Nature of the $\mathrm{Eu}^{2+}$ Luminescence," physica status solidi (b), vol. 55, pp. K131-K134, 1973.

16. G. Särner, M. Richter, and M. Alden, "Investigations of blue emitting phosphors for thermometry," Measurement Science and Technology, vol. 19 , p. 125304,2008

17. B. Fond, C. Abram, and F. Beyrau, "Characterisation of the luminescence properties of BAM:Eu particles as a tracer for thermographic particle image velocimetry" submitted to Applied Physics B, May 2015.

18. R. Turos-Matysiak, M. Grinberg, J. W. Wang, W. M. Yen, and R. S. Meltzer, "Luminescence of BAM under high pressure: the Eu2+ sites," Journal of Luminescence, vol. 122-123, pp. 107-109, 1// 2007.

19. S. Pfadler, F. Dinkelacker, F. Beyrau, and A. Leipertz, "High resolution dual-plane stereo-PIV for validation of subgrid scale models in largeeddy simulations of turbulent premixed flames," Combustion and Flame, vol. 156, pp. 1552-1564, 8// 2009.

20. Hamamatsu Photonics, Photomultiplier Tubes: Basics and Applications, 1 ed., 2007.

21. C. Abram, B. Fond, and F. Beyrau, "High-precision flow temperature imaging using ZnO thermographic phosphor tracer particles", Optics Express 23(15) 19453-19468, 2015. 